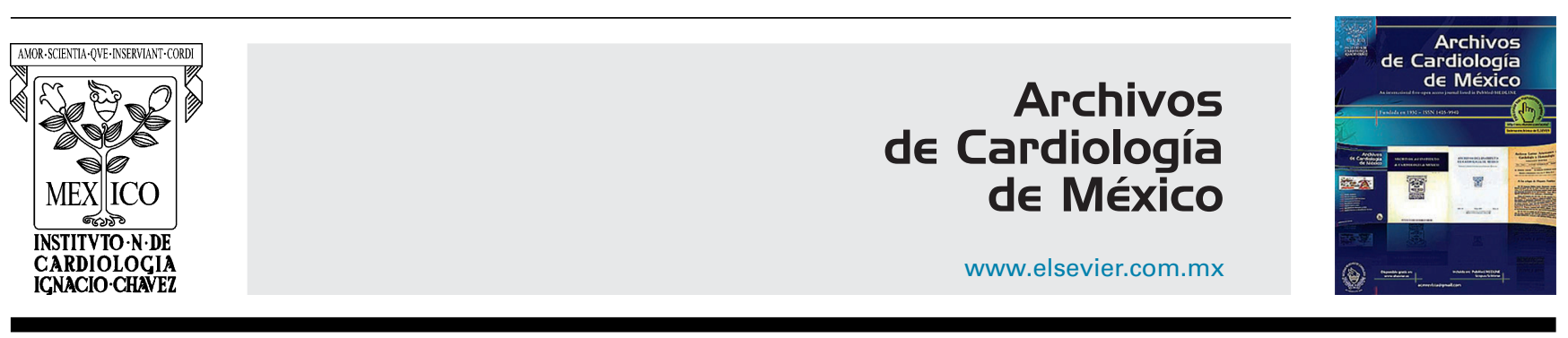

IMAGEN EN CARDIOLOGÍA

\title{
Ritmo acelerado de la unión auriculoventricular
}

\section{Accelerated atrioventricular junctional rhythm}

\section{Juan Ruiz-Garcíaa,b,c,*, Luis Borrego-Bernabé ${ }^{\mathrm{a}, \mathrm{d}}$, Irene Canal-Fontcuberta ${ }^{\mathrm{e}}$ y Eduardo Alegría-Barrero ${ }^{\mathrm{a}, \mathrm{b}, \mathrm{c}}$}

\author{
a Servicio de Cardiología, Hospital Universitario de Torrejón, Torrejón de Ardoz, Madrid, España \\ b Unidad de Cardiología, Hospital Ruber Internacional, Madrid, España \\ c Facultad de Ciencias de la Salud, Universidad Francisco de Vitoria (UFV), Pozuelo de Alarcón, Madrid, España \\ d Servicio de Cardiología, Hospital Clínico San Carlos, Madrid, España \\ e Servicio de Oftalmología, Hospital Universitario de Torrejón, Torrejón de Ardoz, Madrid, España
}

Recibido el 23 de enero de 2017; aceptado el 31 de julio de 2017

Varón de 30 años, fumador, deportista, sin antecedentes médicos personales ni familiares de interés. No sigue tratamiento farmacológico. Es remitido por su médico de cabecera por alteraciones de la repolarización en derivaciones inferiores (fig. 1). Se trata de un ritmo acelerado de la unión auriculoventricular, que cursa con frecuencias cardíacas de entre 70-130 latidos por minuto, y que en adultos es mucho más común que la taquicardia paroxística de la unión, la cual se ve típicamente en niños tras la cirugía de cardiopatías congénitas, a unas frecuencias de 120-220 latidos por minuto ${ }^{1,2}$. Aunque tradicionalmente se ha asociado a intoxicación digitálica o infarto agudo de miocardio ${ }^{1}$, en nuestro caso se trataba de un adulto completamente asintomático, con un ecocardiograma sin cardiopatía estructural significativa. La actividad automática más rápida de las células de la unión condicionaría la supresión del automatismo del nodo sinusal ${ }^{3}$, no debiéndose confundir por tanto con las taquicardias por reentrada de la unión auriculoventricular. Como prueba de ese mecanismo automático, el incremento del tono simpático originado por un pequeño estrés mental logró aumentar la frecuencia basal de disparo (fig. 2A). Por otra parte, la maniobra de Valsalva (fig. 2B) confirmó también la participación del nodo auriculoventricular, consiguiendo transitoriamente el retorno del marcapasos sinusal. En la actualidad, pasados 6 meses de su visita inicial, el paciente continúa practicando ejercicio físico libre de sintomatología cardiovascular, sin haberse detectado otros trastornos del ritmo en el registro electrocardiográfico ambulatorio de $24 \mathrm{~h}$.

\footnotetext{
* Autor para correspondencia. Servicio de Cardiología, Hospital Universitario de Torrejón. Calle Mateo Inurria, s/n. 28850 Torrejón de Ardoz, Madrid (España). Teléfono: +34 916262600.

Correo electrónico: j.ruizgarcia@hotmail.com (J. Ruiz-García).
} 


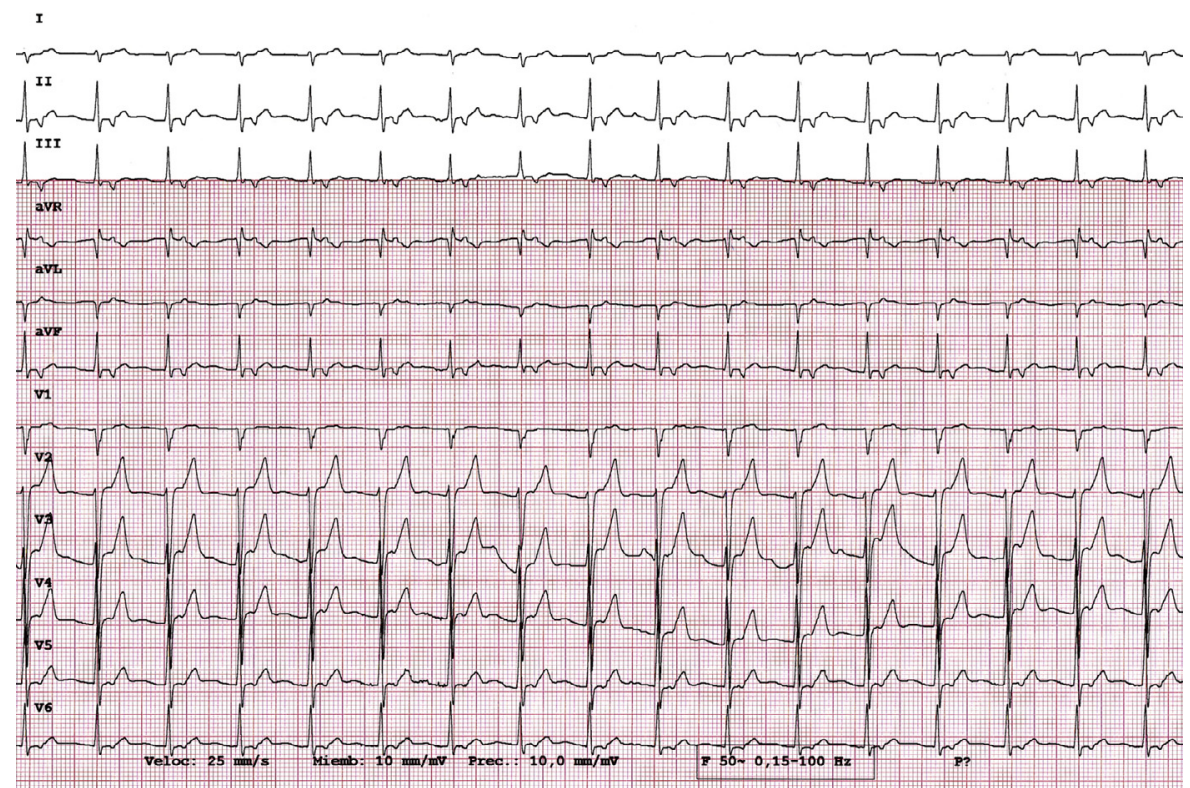

Figura 1 Ritmo basal del paciente.
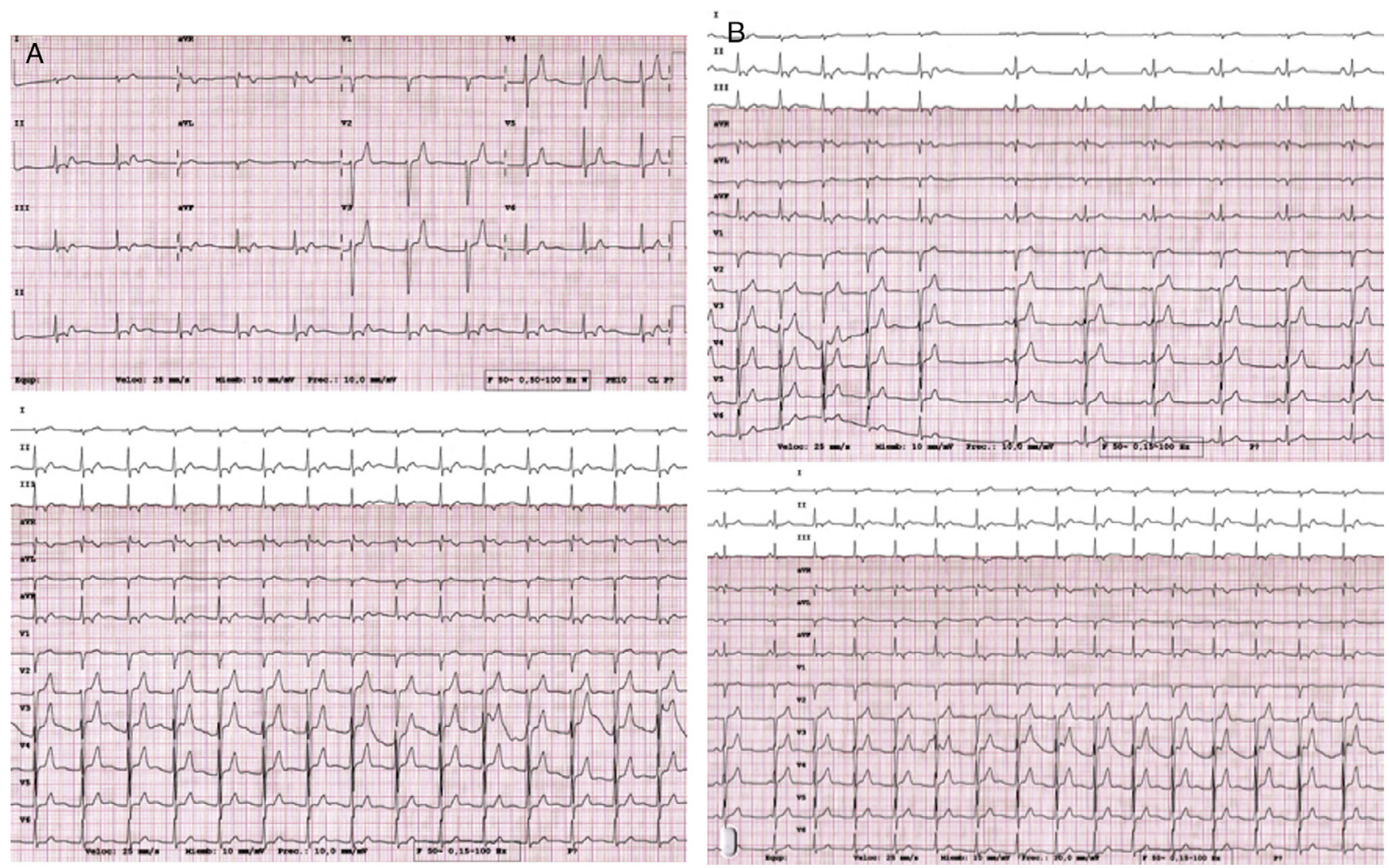

Figura 2 A: Aceleración del ritmo basal del paciente tras provocación de estrés mental. B: Maniobra de Valsalva que induce el retorno transitorio del ritmo sinusal. 


\section{Responsabilidades éticas}

Protección de personas y animales. Los autores declaran que para esta investigación no se han realizado experimentos en seres humanos ni en animales.

Confidencialidad de los datos. Los autores declaran que han seguido los protocolos de su centro de trabajo sobre la publicación de datos de pacientes.

Derecho a la privacidad y consentimiento informado. Los autores han obtenido el consentimiento informado de los pacientes y/o sujetos referidos en el artículo. Este documento obra en poder del autor de correspondencia.

\section{Bibliografía}

1. Page RL, Joglar JA, Caldwell MA, et al. 2015 ACC/AHA/HRS guideline for the management of adult patients with supraventricular tachycardia: A report of the American College of Cardiology/American Heart Association Task Force on Clinical Practice Guidelines and the Heart Rhythm Society. J Am Coll Cardiol. 2016;67:e27-115.

2. Cabrera Duro A, Rodrigo Carbonero D, Galdeano Miranda JM, et al. [The treatment of postoperative junctional ectopic tachycardia]. An Esp Pediatr. 2002;56:505-9.

3. Lee KL, Chun HM, Liem LB, et al. Effect of adenosine and verapamil in catecholamine-induced accelerated atrioventricular junctional rhythm: Insights into the underlying mechanism. Pacing Clin Electrophysiol. 1999;22:866-70. 